\title{
High pressure density of bis(1-alkyl-3-methylimidazolium) tetraisothiocyanatocobaltate ionic liquids: experimental and PC-SAFT with volume-shift modeling
}

Pablo Navarro ${ }^{a}$ André M. Palma ${ }^{a}$, Julián Garcia ${ }^{b}$, Francisco Rodríguez $^{b}$, João A.P. Coutinho ${ }^{a}$, Pedro J. Carvalho ${ }^{a *}$

${ }^{a}$ CICECO - Aveiro Institute of Materials, Department of Chemistry, University of Aveiro, Aveiro, Portugal

${ }^{b}$ Department of Chemical Engineering, Complutense University of Madrid, E-28040 Madrid, Spain.

* To whom correspondence should be addressed. E-mail address: quijorge@ua.pt (Pedro J. Carvalho). 

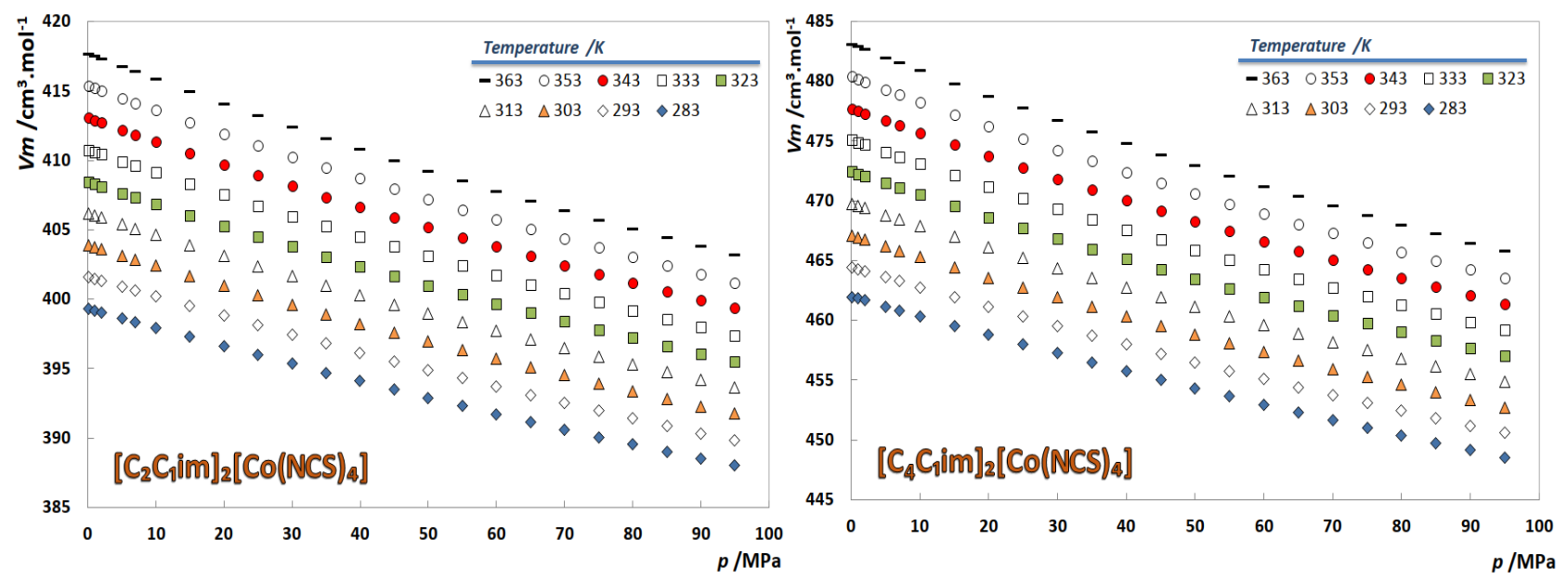

Figure S1. Molar volumes as a function of temperature, pressure, and the studied ILs. 
Table S1. Density as function of temperature and pressure for the $\left[\mathrm{C}_{2} \mathrm{C}_{1} i \mathrm{im}\right]_{2}\left[\mathrm{Co}(\mathrm{NCS})_{4}\right]$.

\begin{tabular}{|c|c|c|c|c|c|c|c|c|c|c|c|c|c|c|c|c|c|}
\hline \multicolumn{18}{|c|}{$T / \mathrm{K}$} \\
\hline \multicolumn{2}{|c|}{283.1} & \multicolumn{2}{|c|}{293.1} & \multicolumn{2}{|c|}{303.1} & \multicolumn{2}{|c|}{313.2} & \multicolumn{2}{|c|}{$323 . \overline{2}$} & \multicolumn{2}{|c|}{333.2} & \multicolumn{2}{|c|}{343.1} & \multicolumn{2}{|c|}{353.1} & \multicolumn{2}{|c|}{363.1} \\
\hline$p / \mathrm{MPa}$ & $\rho / \mathbf{g} \cdot \mathbf{c m}^{-3}$ & $p / \mathbf{M P a}$ & $\rho / \mathrm{g} \cdot \mathrm{cm}^{-3}$ & $p / \mathbf{M P a}$ & $\rho / \mathbf{g} \cdot \mathbf{c m}^{-3}$ & $p / \mathbf{M P a}$ & $\rho / \mathbf{g} \cdot \mathrm{cm}^{-3}$ & $p /$ MPa & $\rho / \mathbf{g} \cdot \mathbf{c m}^{-3}$ & $p / \mathbf{M P a}$ & $\rho / \mathrm{g} \cdot \mathrm{cm}^{-3}$ & p/ MPa & $\rho / \mathbf{g} \cdot \mathrm{cm}^{-3}$ & $p / \mathbf{M P a}$ & $\rho / \mathbf{g} \cdot \mathrm{cm}^{-3}$ & $p / \mathbf{M P a}$ & $\rho / \mathbf{g} \cdot \mathrm{cm}^{-3}$ \\
\hline 0.1 & 1.2861 & 0.1 & 1.2787 & 0.1 & 1.2715 & 0.1 & 1.2644 & 0.1 & 1.2573 & 0.1 & 1.2503 & 0.1 & 1.2433 & 0.1 & 1.2364 & 0.1 & 1.2295 \\
\hline 1.0 & 1.2865 & 1.0 & 1.2791 & 1.0 & 1.2720 & 1.0 & 1.2649 & 1.0 & 1.2578 & 1.0 & 1.2507 & 1.0 & 1.2437 & 1.0 & 1.2369 & 1.0 & 1.2301 \\
\hline 2.0 & 1.2870 & 2.0 & 1.2796 & 2.0 & 1.2725 & 2.0 & 1.2653 & 2.0 & 1.2584 & 2.0 & 1.2513 & 2.0 & 1.2443 & 2.0 & 1.2375 & 2.0 & 1.2306 \\
\hline 5.0 & 1.2884 & 5.0 & 1.2811 & 5.0 & 1.2739 & 5.0 & 1.2668 & 5.0 & 1.2599 & 5.0 & 1.2530 & 5.0 & 1.2460 & 5.0 & 1.2392 & 5.0 & 1.2324 \\
\hline 7.0 & 1.2893 & 7.0 & 1.2821 & 7.0 & 1.2749 & 7.0 & 1.2679 & 7.0 & 1.2609 & 7.0 & 1.2539 & 7.0 & 1.2470 & 7.0 & 1.2403 & 7.0 & 1.2335 \\
\hline 10.0 & 1.2906 & 10.0 & 1.2834 & 10.0 & 1.2763 & 10.0 & 1.2694 & 10.0 & 1.2625 & 10.0 & 1.2553 & 10.0 & 1.2486 & 10.0 & 1.2417 & 10.0 & 1.2351 \\
\hline 15.0 & 1.2928 & 15.0 & 1.2857 & 15.0 & 1.2786 & 15.0 & 1.2717 & 15.0 & 1.2650 & 15.0 & 1.2579 & 15.0 & 1.2513 & 15.0 & 1.2443 & 15.0 & 1.2377 \\
\hline 20.0 & 1.2950 & 20.0 & 1.2879 & 20.0 & 1.2810 & 20.0 & 1.2741 & 20.0 & 1.2673 & 20.0 & 1.2601 & 20.0 & 1.2537 & 20.0 & 1.2469 & 20.0 & 1.2404 \\
\hline 25.0 & 1.2971 & 25.0 & 1.2900 & 25.0 & 1.2831 & 25.0 & 1.2763 & 25.0 & 1.2696 & 25.0 & 1.2627 & 25.0 & 1.2560 & 25.0 & 1.2494 & 25.0 & 1.2430 \\
\hline 30.0 & 1.2991 & 30.0 & 1.2921 & 30.0 & 1.2854 & 30.0 & 1.2787 & 30.0 & 1.2719 & 30.0 & 1.2651 & 30.0 & 1.2583 & 30.0 & 1.2519 & 30.0 & 1.2456 \\
\hline 35.0 & 1.3011 & 35.0 & 1.2943 & 35.0 & 1.2876 & 35.0 & 1.2809 & 35.0 & 1.2741 & 35.0 & 1.2675 & 35.0 & 1.2606 & 35.0 & 1.2543 & 35.0 & 1.2482 \\
\hline 40.0 & 1.3032 & 40.0 & 1.2963 & 40.0 & 1.2896 & 40.0 & 1.2829 & 40.0 & 1.2764 & 40.0 & 1.2695 & 40.0 & 1.2630 & 40.0 & 1.2567 & 40.0 & 1.2505 \\
\hline 45.0 & 1.3052 & 45.0 & 1.2984 & 45.0 & 1.2917 & 45.0 & 1.2851 & 45.0 & 1.2786 & 45.0 & 1.2720 & 45.0 & 1.2655 & 45.0 & 1.2591 & 45.0 & 1.2527 \\
\hline 50.0 & 1.3072 & 50.0 & 1.3005 & 50.0 & 1.2940 & 50.0 & 1.2874 & 50.0 & 1.2808 & 50.0 & 1.2742 & 50.0 & 1.2677 & 50.0 & 1.2612 & 50.0 & 1.2549 \\
\hline 55.0 & 1.3090 & 55.0 & 1.3025 & 55.0 & 1.2959 & 55.0 & 1.2895 & 55.0 & 1.2829 & 55.0 & 1.2764 & 55.0 & 1.2696 & 55.0 & 1.2636 & 55.0 & 1.2572 \\
\hline 60.0 & 1.3110 & 60.0 & 1.3043 & 60.0 & 1.2979 & 60.0 & 1.2914 & 60.0 & 1.2850 & 60.0 & 1.2784 & 60.0 & 1.2718 & 60.0 & 1.2657 & 60.0 & 1.2594 \\
\hline 65.0 & 1.3129 & 65.0 & 1.3063 & 65.0 & 1.2997 & 65.0 & 1.2933 & 65.0 & 1.2870 & 65.0 & 1.2804 & 65.0 & 1.2741 & 65.0 & 1.2677 & 65.0 & 1.2616 \\
\hline 70.0 & 1.3148 & 70.0 & 1.3083 & 70.0 & 1.3017 & 70.0 & 1.2953 & 70.0 & 1.2889 & 70.0 & 1.2824 & 70.0 & 1.2759 & 70.0 & 1.2699 & 70.0 & 1.2637 \\
\hline 75.0 & 1.3166 & 75.0 & 1.3102 & 75.0 & 1.3037 & 75.0 & 1.2972 & 75.0 & 1.2909 & 75.0 & 1.2846 & 75.0 & 1.2779 & 75.0 & 1.2720 & 75.0 & 1.2658 \\
\hline 80.0 & 1.3183 & 80.0 & 1.3122 & 80.0 & 1.3054 & 80.0 & 1.2990 & 80.0 & 1.2928 & 80.0 & 1.2865 & 80.0 & 1.2802 & 80.0 & 1.2742 & 80.0 & 1.2678 \\
\hline 85.0 & 1.3200 & 85.0 & 1.3139 & 85.0 & 1.3074 & 85.0 & 1.3011 & 85.0 & 1.2947 & 85.0 & 1.2886 & 85.0 & 1.2824 & 85.0 & 1.2761 & 85.0 & 1.2701 \\
\hline 90.0 & 1.3219 & 90.0 & 1.3156 & 90.0 & 1.3094 & 90.0 & 1.3029 & 90.0 & 1.2968 & 90.0 & 1.2904 & 90.0 & 1.2843 & 90.0 & 1.2782 & 90.0 & 1.2721 \\
\hline 95.0 & 1.3238 & 95.0 & 1.3175 & 95.0 & 1.3110 & 95.0 & 1.3048 & 95.0 & 1.2987 & 95.0 & 1.2925 & 95.0 & 1.2861 & 95.0 & 1.2802 & 95.0 & 1.2738 \\
\hline
\end{tabular}

Standard uncertainties are: $u(p)=0.01 \mathrm{MPa} ; u(T)=0.1 \mathrm{~K}$. Expanded uncertainty for density $U(\rho)$ is $5 \cdot 10^{-4}{\mathrm{~g} . \mathrm{cm}^{-3}}^{-3}(0.95$ level of confidence). 
Table S2. Density as function of temperature and pressure for the $\left[\mathrm{C}_{4} \mathrm{C}_{1} i \mathrm{im}\right]_{2}\left[\mathrm{Co}(\mathrm{NCS})_{4}\right]$.

\begin{tabular}{|c|c|c|c|c|c|c|c|c|c|c|c|c|c|c|c|c|c|}
\hline \multicolumn{18}{|c|}{$\boldsymbol{T} / \mathrm{K}$} \\
\hline \multicolumn{2}{|c|}{283.16} & \multicolumn{2}{|c|}{293.13} & \multicolumn{2}{|c|}{303.17} & \multicolumn{2}{|c|}{313.17} & \multicolumn{2}{|c|}{323.18} & \multicolumn{2}{|c|}{333.15} & \multicolumn{2}{|c|}{343.14} & \multicolumn{2}{|c|}{353.13} & \multicolumn{2}{|c|}{363.14} \\
\hline$p /$ MPa & $\rho / \mathbf{g} \cdot \mathrm{cm}^{-3}$ & $p / \mathbf{M P a}$ & $\rho / \mathbf{g} \cdot \mathbf{c m}^{-3}$ & $p / \mathbf{M P a}$ & $\rho / \mathbf{g} \cdot \mathrm{cm}^{-3}$ & $p /$ MPa & $\rho / \mathbf{g} \cdot \mathrm{cm}^{-3}$ & $p / \mathbf{M P a}$ & $\rho / \mathbf{g} \cdot \mathbf{c m}^{-3}$ & $p / \mathbf{M P a}$ & $\rho / \mathbf{g} \cdot \mathbf{c m}^{-3}$ & $p /$ MPa & $\rho / \mathrm{g} \cdot \mathrm{cm}^{-3}$ & $p / \mathbf{M P a}$ & $\rho / \mathbf{g} \cdot \mathrm{cm}^{-3}$ & $p / \mathbf{M P a}$ & $\rho / \mathbf{g} \cdot \mathbf{c m}^{-3}$ \\
\hline 0.1 & 1.2331 & 0.1 & 1.2264 & 0.1 & 1.2196 & 0.1 & 1.2126 & 0.1 & 1.2058 & 0.1 & 1.1990 & 0.1 & 1.1924 & 0.1 & 1.1858 & 0.1 & 1.1792 \\
\hline 1.0 & 1.2335 & 1.0 & 1.2269 & 1.0 & 1.2201 & 1.0 & 1.2132 & 1.0 & 1.2063 & 1.0 & 1.1995 & 1.0 & 1.1929 & 1.0 & 1.1863 & 1.0 & 1.1798 \\
\hline 2.0 & 1.2339 & 2.0 & 1.2274 & 2.0 & 1.2205 & 2.0 & 1.2136 & 2.0 & 1.2068 & 2.0 & 1.2001 & 2.0 & 1.1935 & 2.0 & 1.1869 & 2.0 & 1.1803 \\
\hline 5.0 & 1.2353 & 5.0 & 1.2289 & 5.0 & 1.2221 & 5.0 & 1.2152 & 5.0 & 1.2083 & 5.0 & 1.2018 & 5.0 & 1.1951 & 5.0 & 1.1886 & 5.0 & 1.1820 \\
\hline 7.0 & 1.2362 & 7.0 & 1.2297 & 7.0 & 1.2229 & 7.0 & 1.2162 & 7.0 & 1.2094 & 7.0 & 1.2027 & 7.0 & 1.1962 & 7.0 & 1.1896 & 7.0 & 1.1832 \\
\hline 10.0 & 1.2376 & 10.0 & 1.2311 & 10.0 & 1.2243 & 10.0 & 1.2177 & 10.0 & 1.2108 & 10.0 & 1.2042 & 10.0 & 1.1977 & 10.0 & 1.1912 & 10.0 & 1.1848 \\
\hline 15.0 & 1.2398 & 15.0 & 1.2335 & 15.0 & 1.2268 & 15.0 & 1.2201 & 15.0 & 1.2133 & 15.0 & 1.2067 & 15.0 & 1.2004 & 15.0 & 1.1938 & 15.0 & 1.1875 \\
\hline 20.0 & 1.2419 & 20.0 & 1.2356 & 20.0 & 1.2291 & 20.0 & 1.2225 & 20.0 & 1.2158 & 20.0 & 1.2092 & 20.0 & 1.2028 & 20.0 & 1.1965 & 20.0 & 1.1900 \\
\hline 25.0 & 1.2440 & 25.0 & 1.2376 & 25.0 & 1.2313 & 25.0 & 1.2248 & 25.0 & 1.2181 & 25.0 & 1.2116 & 25.0 & 1.2051 & 25.0 & 1.1989 & 25.0 & 1.1926 \\
\hline 30.0 & 1.2459 & 30.0 & 1.2397 & 30.0 & 1.2335 & 30.0 & 1.2269 & 30.0 & 1.2205 & 30.0 & 1.2139 & 30.0 & 1.2075 & 30.0 & 1.2013 & 30.0 & 1.1950 \\
\hline 35.0 & 1.2480 & 35.0 & 1.2418 & 35.0 & 1.2356 & 35.0 & 1.2291 & 35.0 & 1.2228 & 35.0 & 1.2162 & 35.0 & 1.2098 & 35.0 & 1.2036 & 35.0 & 1.1974 \\
\hline 40.0 & 1.2501 & 40.0 & 1.2438 & 40.0 & 1.2377 & 40.0 & 1.2313 & 40.0 & 1.2249 & 40.0 & 1.2183 & 40.0 & 1.2122 & 40.0 & 1.2059 & 40.0 & 1.1999 \\
\hline 45.0 & 1.2519 & 45.0 & 1.2459 & 45.0 & 1.2397 & 45.0 & 1.2333 & 45.0 & 1.2270 & 45.0 & 1.2205 & 45.0 & 1.2144 & 45.0 & 1.2081 & 45.0 & 1.2022 \\
\hline 50.0 & 1.2539 & 50.0 & 1.2480 & 50.0 & 1.2417 & 50.0 & 1.2353 & 50.0 & 1.2291 & 50.0 & 1.2228 & 50.0 & 1.2164 & 50.0 & 1.2105 & 50.0 & 1.2045 \\
\hline 55.0 & 1.2558 & 55.0 & 1.2500 & 55.0 & 1.2436 & 55.0 & 1.2374 & 55.0 & 1.2311 & 55.0 & 1.2249 & 55.0 & 1.2186 & 55.0 & 1.2128 & 55.0 & 1.2067 \\
\hline 60.0 & 1.2576 & 60.0 & 1.2519 & 60.0 & 1.2456 & 60.0 & 1.2394 & 60.0 & 1.2332 & 60.0 & 1.2270 & 60.0 & 1.2207 & 60.0 & 1.2151 & 60.0 & 1.2089 \\
\hline 65.0 & 1.2594 & 65.0 & 1.2538 & 65.0 & 1.2475 & 65.0 & 1.2413 & 65.0 & 1.2351 & 65.0 & 1.2291 & 65.0 & 1.2229 & 65.0 & 1.2169 & 65.0 & 1.2112 \\
\hline 70.0 & 1.2613 & 70.0 & 1.2555 & 70.0 & 1.2494 & 70.0 & 1.2434 & 70.0 & 1.2372 & 70.0 & 1.2310 & 70.0 & 1.2250 & 70.0 & 1.2190 & 70.0 & 1.2132 \\
\hline 75.0 & 1.2632 & 75.0 & 1.2574 & 75.0 & 1.2513 & 75.0 & 1.2452 & 75.0 & 1.2392 & 75.0 & 1.2330 & 75.0 & 1.2270 & 75.0 & 1.2211 & 75.0 & 1.2153 \\
\hline 80.0 & 1.2649 & 80.0 & 1.2591 & 80.0 & 1.2531 & 80.0 & 1.2470 & 80.0 & 1.2410 & 80.0 & 1.2351 & 80.0 & 1.2291 & 80.0 & 1.2231 & 80.0 & 1.2173 \\
\hline 85.0 & 1.2666 & 85.0 & 1.2610 & 85.0 & 1.2548 & 85.0 & 1.2489 & 85.0 & 1.2429 & 85.0 & 1.2369 & 85.0 & 1.2310 & 85.0 & 1.2252 & 85.0 & 1.2193 \\
\hline 90.0 & 1.2684 & 90.0 & 1.2626 & 90.0 & 1.2567 & 90.0 & 1.2506 & 90.0 & 1.2448 & 90.0 & 1.2389 & 90.0 & 1.2328 & 90.0 & 1.2271 & 90.0 & 1.2214 \\
\hline 95.0 & 1.2701 & 95.0 & 1.2644 & 95.0 & 1.2585 & 95.0 & 1.2525 & 95.0 & 1.2465 & 95.0 & 1.2407 & 95.0 & 1.2348 & 95.0 & 1.2291 & 95.0 & 1.2233 \\
\hline
\end{tabular}

Standard uncertainties are: $u(p)=0.02 \mathrm{MPa} ; u(T)=0.1 \mathrm{~K}$. Expanded uncertainty for density $U(\rho)$ is $5 \cdot 10^{-4}{\mathrm{~g} . \mathrm{cm}^{-3}}^{-3}(0.95$ level of confidence). 